\title{
Secreted indole serves as a signal for expression of type III secretion system translocators in enterohaemorrhagic Escherichia coli O157: H7
}

Correspondence

Akihito Yamaguchi

akihito@sanken.osaka-u.ac.jp

Received 8 May 2008

Revised 29 August 2008

Accepted 2 September 2008
Hidetada Hirakawa, ${ }^{1,4}$ Toshio Kodama, ${ }^{3}$ Asuka Takumi-Kobayashi, ${ }^{1,2}$ Takeshi Honda ${ }^{3}$ and Akihito Yamaguchi ${ }^{1,2}$

\author{
${ }^{1}$ Department of Cell Membrane Biology, Institute of Scientific and Industrial Research, Osaka \\ University, Ibaraki, Osaka 567-0047, Japan \\ ${ }^{2}$ Graduate School of Pharmaceutical Science, Osaka University, Suita, Osaka 565-0871, Japan \\ ${ }^{3}$ Research Institute for Microbial Diseases, Osaka University, Suita, Osaka 565-0871, Japan \\ ${ }^{4}$ Department of Microbiology, University of Washington, WA 98195, USA
}

\section{INTRODUCTION}

Many enteric bacteria including Escherichia coli produce indole from tryptophan, a reaction that is catalysed by tryptophanase (TnaA). Indole is exported by the AcrEFTolC multidrug efflux system, and accumulates in culture media during the stationary phase (DeMoss \& Moser, 1969; Kawamura-Sato et al., 1999; Wang et al., 2001). TnaA is strongly induced under alkaline conditions (Blankenhorn et al., 1999) and is regulated by catabolite repression (Deeley \& Yanofsky, 1982). Therefore, enterobacteria may be exposed to high concentrations of indole when they infect alkaline regions in the body such as the pancreatic duct. To elucidate the behaviour of enteric bacteria at their site of infection and in their habitat, it is therefore important to understand the regulatory mechanism and the roles of indole signalling.

We previously reported that indole induces multidrug resistance as a result of its ability to stimulate transcription of E. coli drug exporter genes ( $m d t E F$, acrD, $m d t A B C$, acrEF, cusABC, emrKY and yceL) through multiple regulatory pathways [BaeS-BaeR and CpxA-CpxR (the bacterial two-component signal transduction pathway) and

Abbreviations: $A / E$, attaching and effacing; EHEC, enterohaemorrhagic Escherichia coli; LEE, locus of enterocyte effacement; T3SS, type III secretion system.
RpoS-Hfq[GadY]-GadX (a two-component signal transduction system-independent regulatory cascade)] during stationary phase (Hirakawa et al., 2005; Kobayashi et al., 2006). Other studies have shown that indole controls biofilm formation and the expression of genes ( $g a b T$, astD and $t n a A B$ ) for amino acid metabolism in E. coli K-12 (Di Martino et al., 2003; Lee et al., 2007b; Wang et al., 2001).

Enterohaemorrhagic E. coli (EHEC) causes severe infectious diseases, non-bloody diarrhoea, haemorrhagic colitis and haemolytic uraemic syndrome, which can lead to the death of the patient (Karmali, 1989; Nataro \& Kaper, 1998). EHEC adheres to epithelial cells, triggering the formation of hallmark lesions called attaching and effacing (A/E) lesions at an early stage of infection (Nataro \& Kaper, 1998). A/E lesions are characterized by the localized destruction of brush border microvilli, intimate attachment of the organism to the host cell membrane and formation of an underlying actin-rich structure in the host cell. The capacity to form $\mathrm{A} / \mathrm{E}$ lesions is conferred by a type III secretion system (T3SS) and the proteins that it secretes (translocators and effectors), which are gene products of the locus of enterocyte effacement (LEE), a $36 \mathrm{kbp}$ chromosomal pathogenicity island carrying 41 ORFs clustered in five major operons (LEE1 to LEE5) (McDaniel \& Kaper, 1997). To counter EHEC pathogenesis, it is therefore 
important to identify the signals that induce secretion and/or expression of the secreted proteins and to elucidate the mechanism of regulation of LEE expression and type III secretion-related protein secretion under conditions similar to those at sites of infection.

When EHEC adhere to epithelial cells, secreted Tir (an LEE5 gene product) is translocated into the host cell membranes, where it serves as the receptor for intimin, an extracellular bacterial adhesin. EHEC contact host cells via the interaction of intimin and Tir receptor. EspA and EspB (LEE4 gene products) are translocators required for the targeting of Tir. Therefore, these proteins are essential for T3SS-dependent pathogenicity of EHEC.

The recent sequencing of the complete EHEC O157:H7 genome has revealed an ORF homologous to tnaA that encodes tryptophanase in E. coli K-12. To investigate roles of indole in the T3SS-dependent pathogenicity of EHEC, we examined effects of tnaA deletion and of adding indole to a tnaA deletion mutant on the secretion and production of EspA, EspB and Tir and on A/E lesion production. We found that indole enhances secretion and production of EspA/B and also production of type III-dependent A/E lesions.

\section{METHODS}

Bacterial strains, host cells and plasmids. EHEC O157 : H7 Sakai (RIMD 0509952) (Hayashi et al., 2001), its derivative strains, and the plasmids used in this study are described in Table 1. Bacteria were grown in LB broth. Gene-deletion mutants of EHEC except for the $h f q$ single-deletion mutant were constructed by a previously described pKO3-based gene-replacement method (Link et al., 1997). Basically, all the genes targeted for inactivation were replaced by a $33 \mathrm{bp}$ linker sequence that retained the start and stop codons. The No and Co primers described in Table 2 anneal at about $500 \mathrm{bp}$ upstream of the start codon and about $500 \mathrm{bp}$ downstream of the stop codon, respectively. To verify the genedeletion mutations, we performed PCR with No and Co primers, and the length of the respective PCR fragments was compared in the wild-type and the gene-deletion mutant. For construction of the $h f q$ single-deletion mutant, the chloramphenicol-resistance cassette was inserted into the internal region of the $h f q$ gene. A DNA fragment including the $300 \mathrm{bp}$ upstream and $48 \mathrm{bp}$ of coding region of $h f q$ was cloned upstream of the chloramphenicol resistance gene in the suicide vector pYAK1 (Kodama et al., 2002). After homologous recombination, the inactivation of $h f q$ was established. HeLa cells (RIKEN) were grown in Dulbecco's modified Eagle's medium (DMEM, Sigma) containing $10 \%$ fetal bovine serum (FBS, Sigma) and $100 \mathrm{mg}$ gentamicin $\mathrm{l}^{-1}$ (Sigma) at $37{ }^{\circ} \mathrm{C}$ under $5 \% \mathrm{CO}_{2}$ in air. Before infection, the HeLa cells were washed with PBS before the FBS and gentamicin-free DMEM were added.

Table 1. Bacterial strains and plasmids used in this study

\begin{tabular}{|c|c|c|}
\hline Strain or plasmid & Relevant characteristics & Reference or source \\
\hline \multicolumn{3}{|l|}{ E. coli strains } \\
\hline O157: H7 Sakai & Wild-type EHEC O157:H7 (RIMD 0509952) & Hayashi et al. (2001) \\
\hline 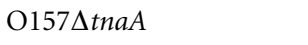 & & This study \\
\hline $\mathrm{O} 157 \Delta l a c Z I$ & & This study \\
\hline 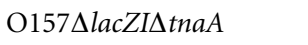 & & This study \\
\hline O157 & & This study \\
\hline 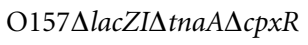 & & This study \\
\hline 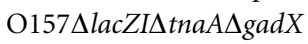 & & This study \\
\hline 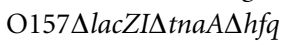 & & This study \\
\hline 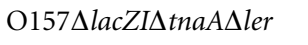 & & This study \\
\hline $\mathrm{O} 157 \Delta e s c N$ & & Cantarelli et al. (2007) \\
\hline $\mathrm{O} 157 \Delta e s c N \Delta t n a A$ & & This study \\
\hline $\mathrm{O} 157 \Delta h f q: \mathrm{Cm}^{\mathrm{r}}$ & & This study \\
\hline \multicolumn{3}{|l|}{ Plasmids } \\
\hline pKO3 & $\operatorname{rep}(\mathrm{Ts}), \mathrm{Cm}^{\mathrm{r}}, s a c B^{+}$ & Link et al. (1997) \\
\hline pNN387 & $\begin{array}{l}\text { Single-copy vector, } \mathrm{Cm}^{\mathrm{r}} \text {, NotI-HindIII cloning site upstream } \\
\text { of promoterless lacZY }\end{array}$ & Elledge \& Davis (1989) \\
\hline pNNLEE1-P & pNN387 (LEE1 gene promoter-lacZ) & This study \\
\hline pNNLEE2-P & pNN387 (LEE2 gene promoter-lacZ) & This study \\
\hline pNNLEE3-P & pNN387 (LEE3 gene promoter-lacZ) & This study \\
\hline pNNLEE4-P & pNN387 (LEE4 gene promoter-lacZ) & This study \\
\hline pNNLEE5-P & pNN387 (LEE5 gene promoter-lacZ) & This study \\
\hline pTrc99A & $\begin{array}{l}\text { Expression vector, } \mathrm{Ap}^{\mathrm{r}} \text {, multiple cloning site downstream } \\
\text { of } \operatorname{trc} \text { promoter }\end{array}$ & Amersham Pharmacia Biotech \\
\hline pTrc99tnaA & Expresses TnaA from $\operatorname{trc}$ promoter & This study \\
\hline pYAK1 & R6Kori, $\mathrm{Cm}^{\mathrm{r}}$, sacB ${ }^{+}$ & Kodama et al. (2002) \\
\hline pUC19hfq & Expresses $\mathrm{Hfq}$ from native promoter & This study \\
\hline
\end{tabular}


Table 2. Oligonucleotides used for plasmid construction, and verification of gene replacement

Bold type indicates the restriction enzyme sites.

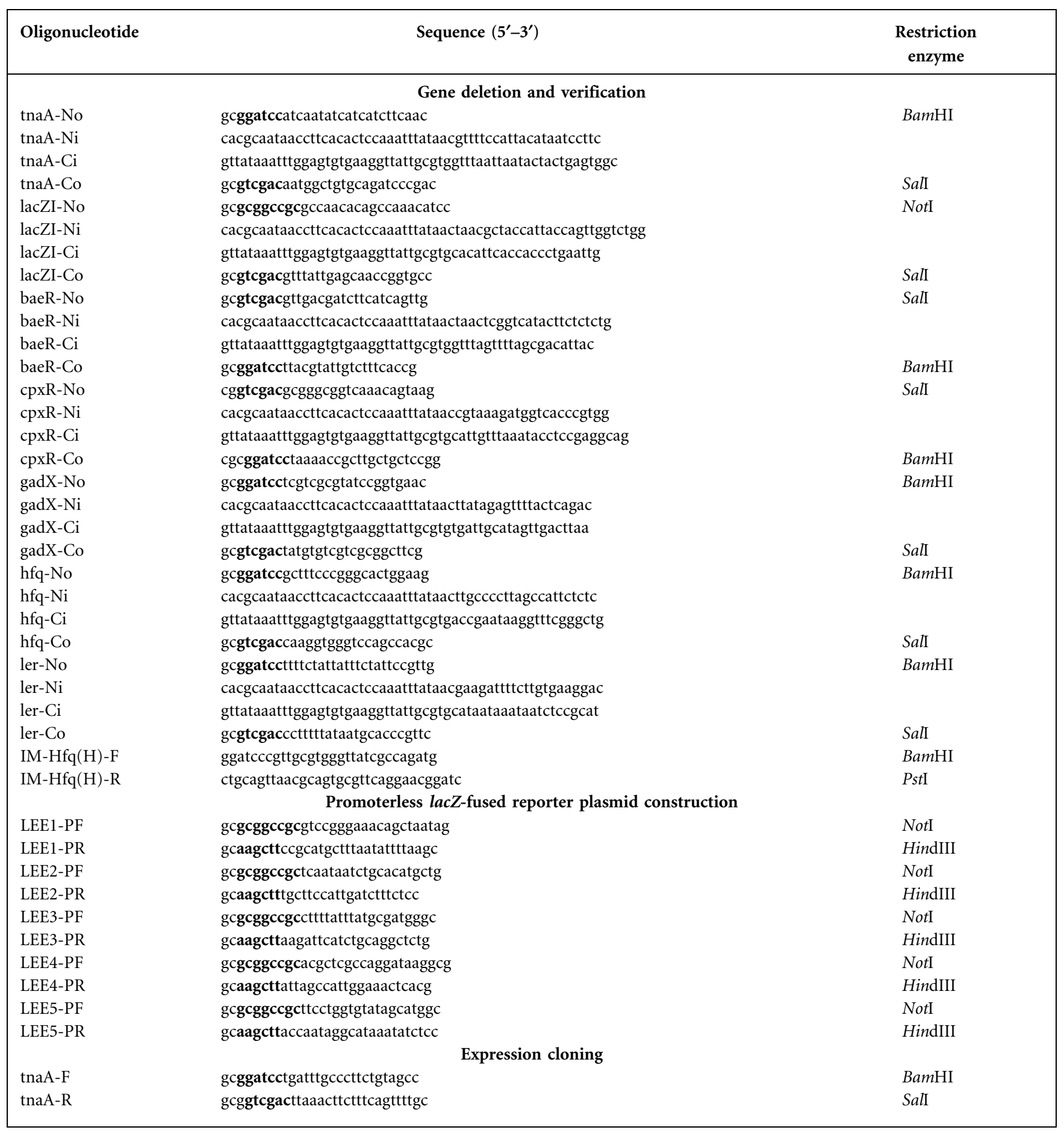

A tnaA expression plasmid was constructed as follows. The tnaA gene including a sequence $220 \mathrm{bp}$ upstream of the start codon was cloned into pTrc99A by using the primers listed in Table 2. This plasmid was named pTrc99tnaA. We expected that TnaA would be expressed under control of the native promoter and IPTG-inducible trc promoter. In the presence of excess IPTG, the growth rate was reduced, but addition of $10 \mu \mathrm{M}$ IPTG did not affect the growth. Therefore, TnaA expression studies were done on cells grown with
$10 \mu \mathrm{M}$ IPTG. In the $\beta$-galactosidase assay, $\Delta l a c Z I$ and derivative strains lacking the chromosomal lac $Z$ gene were used to eliminate the effects of endogenous $\beta$-galactosidase.

For construction of a $h f q$ expression plasmid, the $h f q$ gene including native promoter was amplified by PCR with hfq-F (gccggatcccactgttagtggg) and hfq-R (gccaagcttacagcccgaaaccttattcgg) primers. The fragment was ligated into pUC19 vector. 
Indole production assay. The extracellular indole concentration was determined by a previously described HPLC method (Kobayashi et al., 2006). EHEC and its derivative strains were grown at $37^{\circ} \mathrm{C}$ in LB broth and pelleted by centrifugation at $20000 \mathrm{~g}$. The resulting supernatants were extracted twice with ethyl acetate and the extracts were loaded on to a Symmetry $\mathrm{C}_{18}$ column $(5 \mu \mathrm{m}, 4.6 \times 150 \mathrm{~mm}$ : Waters $)$ attached to an L2130 HPLC system (Hitachi). The samples were eluted with acetonitrile/ $\mathrm{H}_{2} \mathrm{O}(1: 1, \mathrm{v} / \mathrm{v})$ at a flow rate of $0.8 \mathrm{ml} \mathrm{min} \mathrm{m}^{-1}$; the indole peak was detected by its absorbance at $276 \mathrm{~nm}$ and its identity was confirmed by correspondence to the elution time for pure indole. The indole concentration was calculated from the ratio of the area of the detected peak to that of a standard peak.

Detection of EspA and EspB. Cells were grown at $37{ }^{\circ} \mathrm{C}$ for $8 \mathrm{~h}$ (stationary phase) and separated by centrifugation and filtration. Secreted proteins were precipitated from the supernatants with $10 \%$ TCA and dissolved in SDS sample buffer. Cell pellets were dissolved in SDS sample buffer and the solutions were boiled. The samples were analysed by $10 \%$ SDS-PAGE. EspA and EspB were detected by Western blotting with an EspA-specific polyclonal antibody and EspB-specific antiserum as described previously (Cantarelli et al., 2007; Kodama et al., 2002). Protein bands of EspA and EspB were visualized on a LAS-3000 Luminescent Image Analyser (Fujifilm) or X-ray film, and they were quantified by Image J software (National Institutes of Health).

Detection of A/E lesions by fluorescence microscopy. HeLa cells were grown on glass coverslips and infected for $4 \mathrm{~h}$ with $1 \mu \mathrm{l}$ (3.2-3.4 $\times 10^{6}$ bacterial cells) EHEC from an $8 \mathrm{~h}$ shaking culture (stationary phase) in LB broth. After infection, the cells were washed five times with PBS, fixed with $3 \%$ paraformaldehyde for $15 \mathrm{~min}$, and permeabilized with $0.1 \%$ Triton X-100 in PBS. F-actin was stained with rhodamine/phalloidin ( $1 \mathrm{U} \mathrm{ml}^{-1}$, Molecular Probes) and the HeLa cell nuclei were visualized with Hoechst $33258\left(1 \mu \mathrm{g} \mathrm{ml}{ }^{-1}\right.$, Molecular Probes). The mounted samples were observed by fluorescence microscopy, and the number of A/E/ lesions formed on 100 randomly selected cells was counted. HeLa cells were counted in three microscopic fields; \pm values indicate the range of $\mathrm{A} / \mathrm{E}$ lesion numbers in three microscopic fields

Construction of LEE gene reporter plasmids. The reporter plasmids were constructed as follows. DNA fragments containing the promoter region $[513 \mathrm{bp}$ upstream of the start codon for the first gene (ler of LEE1 and sepZ of LEE2) of the LEE1 and LEE2 operons and 506, 516 and 395 bp upstream of the start codon for the first gene (Ecs4570 of LEE3, sepL of LEE4 and tir of LEE5) of the LEE3, LEE4 and LEE5 operons, respectively] were amplified by PCR by using the primers listed in Table 2. These DNA fragments were cloned in front of the promoterless lacZ reporter gene in the single-copy pNN387 vector (Elledge \& Davis, 1989). The resulting plasmids were introduced into EHEC and its derivative strains for $\beta$-galactosidase assays.

Reporter gene assays. To determine the effects of indole and TnaA on the transcription of various reporter constructs, each bacterial strain was grown for $8 \mathrm{~h}$ (stationary phase) or $2 \mathrm{~h}$ (exponential phase) at $37^{\circ} \mathrm{C}$ in LB broth containing indole and chloramphenicol. $\beta$-Galactosidase activity was assayed in cell lysates using ONPG as the substrate (Miller, 1992).

\section{RESULTS}

\section{Indole production by EHEC 0157 : H7 Sakai and the tnaA deletion mutant}

To examine indole production by EHEC O157:H7 Sakai and the tnaA deletion mutant (Ecs4645), extracellular

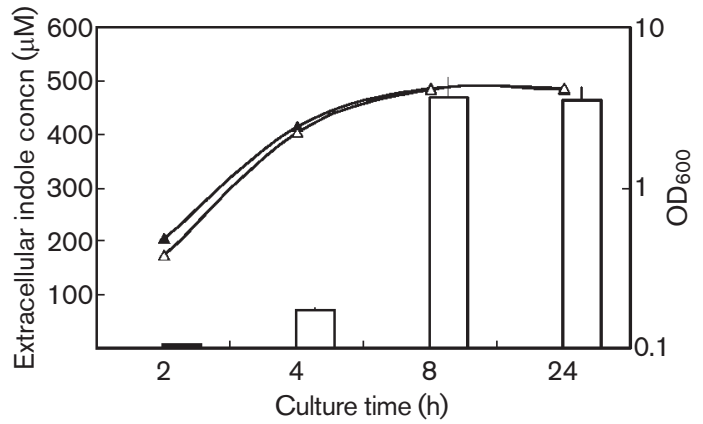

Fig. 1. Indole production (bars) and growth (triangles) of wild-type EHEC (open symbols) and the tha $A$ deletion mutant (solid symbols) cultured in LB medium. The extracellular indole concentrations were measured by HPLC, as described in Methods, after 2, 4, 8, and $24 \mathrm{~h}$ of culture. The EHEC cell density was determined as $\mathrm{OD}_{600}$. At least three independent experiments were performed in each case.

concentrations were measured as described in Methods. When wild-type cells were grown for $8 \mathrm{~h}$ (stationary phase) in LB medium, the extracellular concentration of indole reached a maximum value of $465 \mu \mathrm{M}$ and did not increase on further incubation (Fig. 1). In contrast, the tnaA deletion mutant produced no detectable indole. Growth of the $\Delta t n a A$ strain was the same as that of the wild-type (Fig. 1). We also examined the indole productivity of EHEC grown in tissue cell culture medium (DMEM). However, wild-type EHEC hardly produced indole (extracellular concentration was less than $10 \mu \mathrm{M}$; data not shown). Therefore, to investigate the effect of tnaA deletion and indole addition on EHEC pathogenicity, EHEC and its tnaA mutant were cultured in LB medium for $8 \mathrm{~h}$ in this study.

\section{Effects of indole on the secretion and production of T3SS-dependent EspA and EspB}

When EHEC infect epithelial cells, Tir secreted by EHEC is translocated to the plasma membrane of the host cell by EspA and EspB. Therefore, these proteins play central roles in T3SS-mediated EHEC pathogenicity. To examine the relationship between EHEC pathogenicity and indole, we assessed the effects of the tnaA deletion and indole addition on the secretion and production of EspA, EspB and Tir by immunoblotting with anti-EspA antibody, anti-EspB antiserum and anti-Tir antibody. The secretion of EspA and EspB was greatly decreased in the tnaA deletion mutant (Fig. 2) whereas that of Tir was hardly affected (data not shown). The tnaA expression plasmid pTrc99tnaA restored most of the EspA and EspB secretion in the $\Delta$ tnaA strain (Fig. 2). To determine whether the reduction of EspA/B proteins caused by tnaA deletion is due to the loss of indole, $\Delta$ tnaA was cultured in the presence and absence of indole. The secretion of EspA and EspB was increased to the wild-type level by addition of 125 or $250 \mu \mathrm{M}$ indole (Fig. 2). Similarly, the amount of 


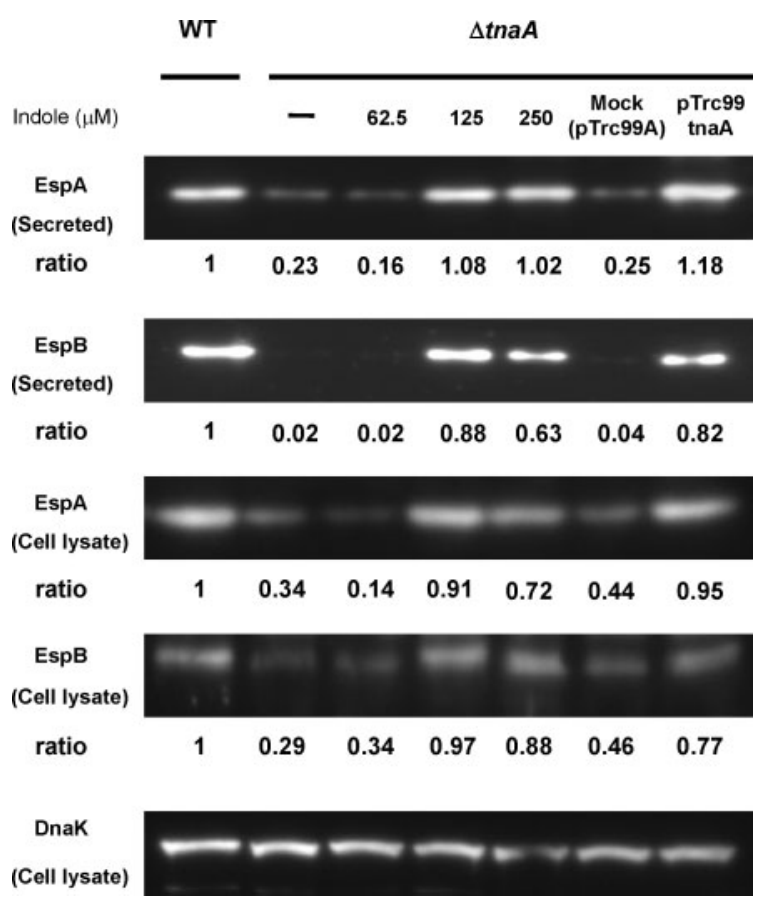

Fig. 2. Secretion and production of $E s p A$ and $E s p B$ in wild-type and $\triangle t n a A$ EHEC $8 \mathrm{~h}$ stationary-phase cultures. EspA and EspB were detected by Western blotting as described in Methods. Wild-type, $\Delta t n a A$ (in the presence or absence of indole), $\Delta t n a A$ harbouring the $\mathrm{p}$ Trc99A vector (mock) (containing $0.01 \mathrm{mM}$ IPTG) and $\Delta$ tnaA harbouring the pTrc99tnaA plasmid (containing $0.01 \mathrm{mM}$ IPTG) were grown for $8 \mathrm{~h}$ and secreted and intracellular EspA/B were measured. As a control, intracellular DnaK was detected by means of a DnaK-specific antibody (Calbiochem). The respective protein bands were visualized on a LAS-3000 Luminescent Image Analyser (Fujifilm), and quantified by using Image J software; values indicate the ratio against the parent strain.

intracellular EspA/B was also decreased by the tnaA deletion, whereas it was restored by the addition of 125 or $250 \mu \mathrm{M}$ indole (Fig. 2). The effect of tnaA deletion and indole addition on EspA/B secretion and production in DMEM was also examined. As in the case of LB medium, indole addition of $125 \mu \mathrm{M}$ increased the amount of extracellular EspA/B by a factor of 1.5 (data not shown). However, the tnaA deletion did not decrease the amount of EspA/B because EHEC cultured in DMEM produces indole poorly. In contrast to the case of extracellular EspA/B, the amount of intracellular EspA/B hardly increased in response to indole addition while the intracellular EspA/ $B$ level in DMEM was higher than that in LB (data not shown). Therefore, the effect of indole may be masked in DMEM.

To determine whether indole stimulates only the production of EspA/B, or both the production and secretion of these proteins, the effect of indole on a T3SS mutant was investigated. The T3SS mutant used, O157 $\Delta e s c N$, lacks the gene encoding the ATPase of the T3SS. Thus O157 $\Delta e s c N$

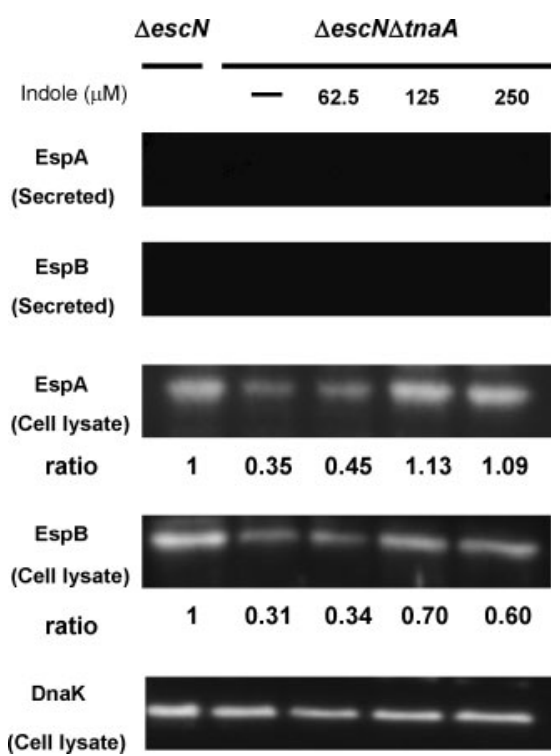

Fig. 3. Secretion and production of EspA and EspB in $\Delta e s c N$ and $\Delta$ escN $\Delta$ tna $A$ EHEC strains cultured in LB medium for $8 \mathrm{~h}$ (stationary phase). $\Delta e s c N$ and $\Delta \operatorname{escN} \Delta \operatorname{tna} A$ (in the presence or absence of indole) were cultured and extracellular and intracellular EspA and EspB were detected. As a negative control, intracellular DnaK was detected by means of a DnaK-specific antibody (Calbiochem). The respective protein bands were visualized and quantified as described for Fig. 2.

cannot secrete translocators or effectors (Cantarelli et al., 2007). In the $\operatorname{esc} N$ deletion mutant, intracellular levels of EspA/B were decreased by the tnaA deletion, and increased by the addition of indole (Fig. 3). However, the influence of the tnaA deletion and indole addition in $\mathrm{O} 157 \Delta e s c N$ was less than that in the parent strain, indicating that indole stimulates not only EspA/B production but also secretion (Fig. 3).

\section{Effect of indole on LEE promoter activity in EHEC}

Type III secretion-related genes are located in the locus of enterocyte effacement (LEE), a pathogenicity island that comprises five major operons (LEE1 to LEE5). Among them, EspA and EspB are encoded on the LEE4 operon. To examine whether indole induces the expression of LEE4 genes, we measured the promoter activity by using the reporter in the wild-type strain and the tnaA deletion mutant in the presence or absence of indole. The promoter activity of LEE4 in the tnaA deletion mutant was lower than that in the wild-type strain, and was restored by the addition of $125 \mu \mathrm{M}$ indole (Fig. 4a). The promoter activity of LEE4 was increased up to fourfold by $2 \mathrm{mM}$ indole. We also measured the promoter activity of other $L E E$ genes. Activity of the $L E E 1$ promoter was reduced by the tnaA deletion; however, activity was not restored by the addition of indole at concentrations of less than $500 \mu \mathrm{M}$. In the presence of $2 \mathrm{mM}$ indole, the activity was increased twofold (data not shown). The activity of LEE3 
(a)

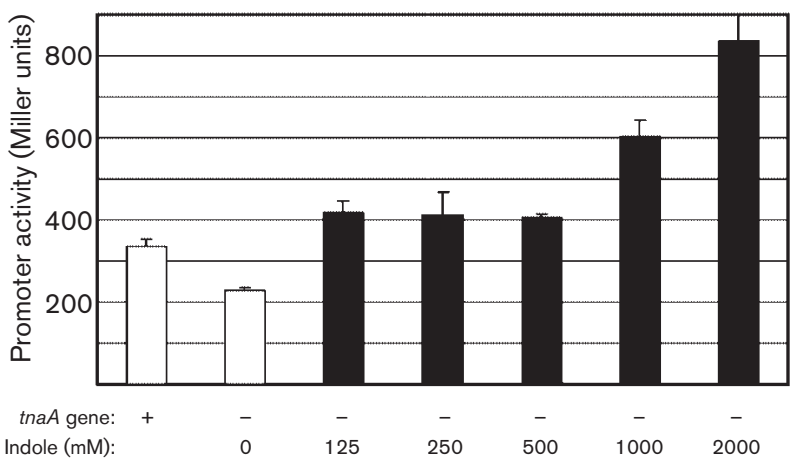

(b)

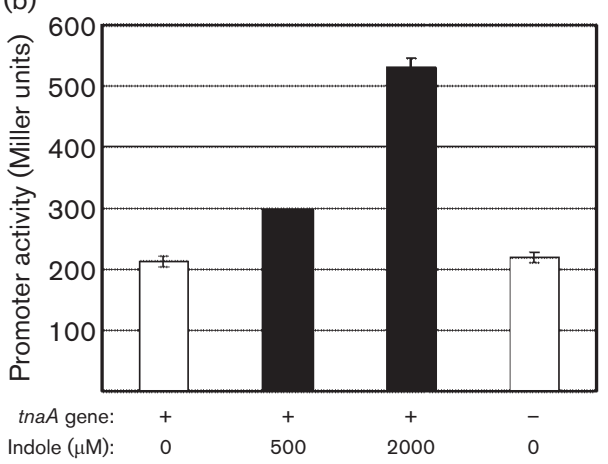

(c)

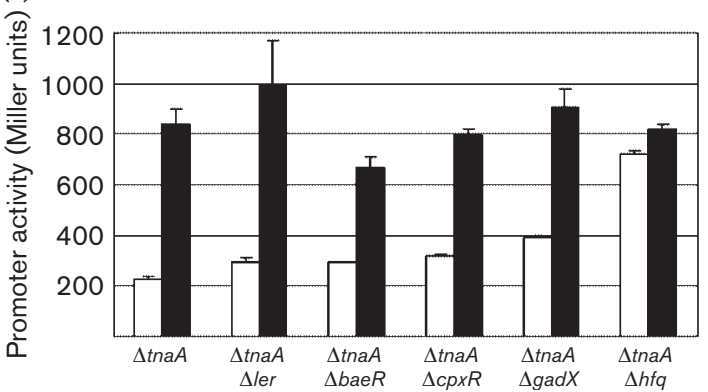

Fig. 4. $(\mathrm{a}, \mathrm{b})$ Effect of indole and tha $A$ gene deletion on $L E E 4$ promoter activity. (a) Wild-type $(\Delta / a c Z I)$ and $\Delta t n a A(\Delta / a c Z I \Delta t n a A)$ EHEC were grown to stationary phase in LB medium with (solid bars) or without (open bars) indole. (b) Wild-type ( $\Delta / a c Z I)$ and $\Delta$ tnaA ( $\Delta / a c Z I \Delta t n a A)$ EHEC cells were grown to exponential phase with (solid bars) or without (open bars) indole. (c) Effects of deletion of ler, baeR, cpxR, gadX and $h f q$ on indole-induced expression of $L E E 4$. The respective mutant strains were grown to stationary phase with (solid bars) or without (open bars) $2 \mathrm{mM}$ indole. The $\beta$-galactosidase activities of the lac $Z$ fusion of the LEE4 promoter were measured. At least three independent experiments were performed in each case.

was also decreased by the tnaA deletion, and increased by the addition of $500 \mu \mathrm{M}$ indole. However, these effects were modest (data not shown). The activities of the LEE2 and LEE5 promoters were not affected by either tnaA deletion or the addition of indole (data not shown).
We also examined whether the LEE4 promoter is stimulated by indole in the wild-type background. TnaA is induced in stationary phase. Therefore, the wild-type and $\Delta$ tnaA strains were cultured to exponential phase (for $2 \mathrm{~h}$ ) in the presence or absence of indole to avoid the effect of intrinsic indole produced by wild-type (Fig. 1), and the activities of the LEE4 promoter were measured. As expected, deletion of tnaA did not reduce activity of the LEE4 promoter, and addition of $500 \mu \mathrm{M}$ or $2 \mathrm{mM}$ indole efficiently enhanced it (Fig. 4b). We believe the primary effect of indole on $L E E$ gene transcription is at the LEE4 promoter.

Next, we investigated the mechanism of regulation of LEE4 expression by indole. We previously reported that the induction of drug exporter genes by indole depends on BaeSR/CpxAR (a two-component signal transduction system in E. coli), GadX (an acid-induced regulatory protein) and Hfq (sRNA chaperone protein) (Kobayashi et al., 2006; Hirakawa et al., 2005). It has also been reported that Ler is a positive regulator of LEE genes in EHEC (Elliott et al., 2000). Therefore, BaeSR, CpxAR, GadX, Hfq and Ler are possible indole signal-transducing mediators in the induction of LEE4 expression. Appropriate deletion mutants were constructed in the $\triangle t n a A$ background, and the effects of indole on LEE4 promoter activity were examined. As shown in Fig. 4(c), LEE4 expression was higher in the $h f q$ deletion mutant $(\Delta l a c Z I \Delta t n a A \Delta h f q)$ than in the $h f q$ intact strain $(\Delta l a c Z I \Delta t n a A)$, and it was not significantly induced by indole (1.1-fold induction). The levels of induction by indole in the other mutants were almost the same as in the parent strain (Fig. 4c). To examine the effect of $h f q$ deletion on EspA/B (gene products of LEE4) production and the sensitivity to indole, levels of secreted and intracellular EspA/B were determined when these mutant strains were cultured in the presence and absence of exogenous indole. The $h f q$ deletion mutant $(\Delta l a c Z I \Delta t n a A \Delta h f q)$ produced large amounts of EspA/B even in the absence of exogenous indole. However, levels of neither secreted EspA/B nor intracellular EspA/B were increased by the addition of indole (Fig. 5). These observations are consistent with the results of the promoter activity measurements. The results indicate that Hfq represses the expression of LEE4 while it is responsible for the induction of LEE4 by indole.

Since the effect of $h f q$ deletion on EspA/B secretion and production had been examined in the tnaA deletion background, we tested whether a single $h f q$ mutant also increases EspA/B secretion and production. A large amount of intracellular and extracellular EspA and EspB was detected in the $\Delta h f q: \mathrm{Cm}$ strain carrying pUC19 vector. However, when the $h f q$ expression plasmid (pUC19hfq) was introduced, the amount of EspA and EspB was decreased to about the wild-type level (Fig. 6).

\section{Effects of indole on the formation of A/E lesions in HeLa cells}

Because the secretion and production of $\mathrm{EspA} / \mathrm{B}$ are stimulated by indole, T3SS-dependent EHEC pathogenicity, 


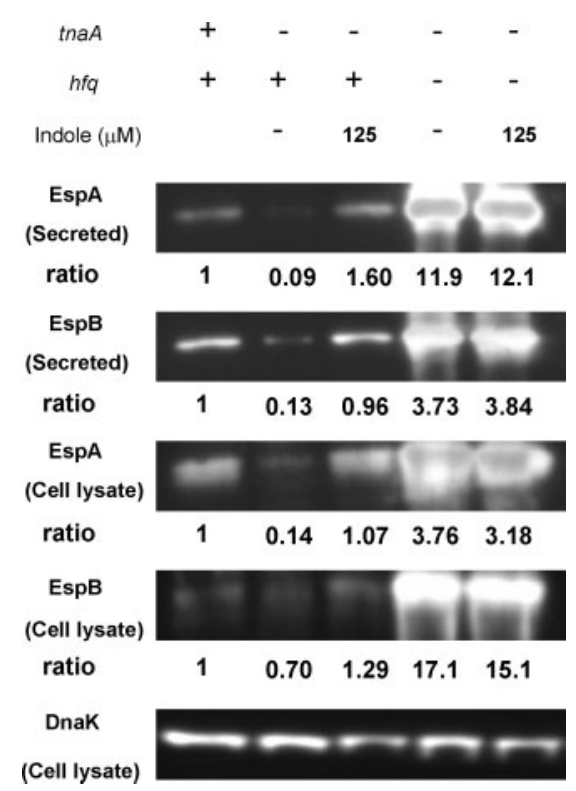

Fig. 5. Secretion and production of EspA and EspB in $\Delta / a c Z I$, $\Delta / a c Z I \Delta t n a A$ and $\Delta / a c Z I \Delta$ tnaA $\Delta h f q$ EHEC. Strains were cultured in LB medium for $8 \mathrm{~h}$ (stationary phase) in the presence or absence of indole, and levels of secreted and intracellular EspA and EspB were detected. As a negative control, intracellular DnaK was detected by means of a DnaK-specific antibody (Calbiochem). The respective protein bands were visualized and quantified as described for Fig. 2.

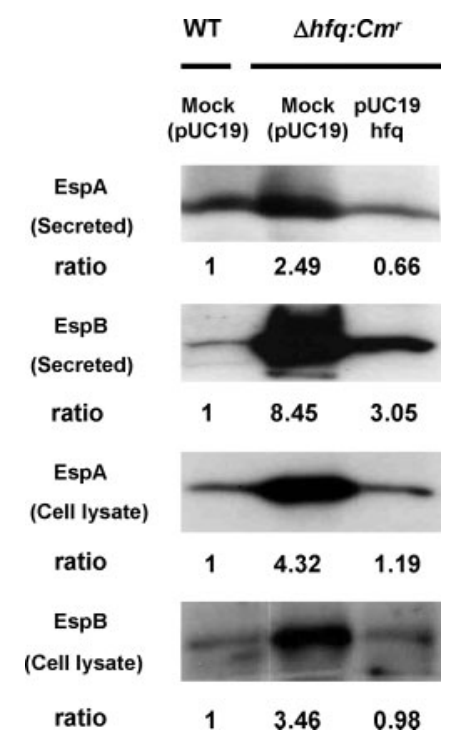

Fig. 6. Secretion and production of EspA and EspB in wild-type and $h f q$ single-mutant $\left(\Delta h f q: \mathrm{Cm}^{r}\right)$ EHEC strains cultured in LB medium for $8 \mathrm{~h}$ (stationary phase). Wild-type and $\Delta h f q: \mathrm{Cm}^{\mathrm{r}}$ harbouring the pUC19 vector (mock), and $\Delta h f q: \mathrm{Cm}^{r}$ harbouring the pUC19hfq ( $h f q$ expression) plasmid were grown for $8 \mathrm{~h}$ and secreted and intracellular EspA/B were measured. The respective protein bands were visualized on X-ray film, and quantified by using Image J software; values indicate ratio against the parent strain. which is characterized by accumulation of actin in the host cells (A/E lesions), may also be stimulated by indole. Therefore, the effects of tnaA deletion and the addition of indole on the formation of $\mathrm{A} / \mathrm{E}$ lesions in HeLa cells were examined. HeLa cells were infected for $4 \mathrm{~h}$ with stationaryphase wild-type EHEC or with indole-treated or untreated $\Delta$ tnaA mutant. The infected cells were fixed and stained with rhodamine-labelled phalloidin and Hoechst 33258 to identify actin and nuclei, respectively. The $\mathrm{A} / \mathrm{E}$ lesions, which are characterized by accumulated F-actin, were then counted. When the HeLa cells were infected with wild-type EHEC, $14 \pm 3 \mathrm{~A} / \mathrm{E}$ lesions per $100 \mathrm{HeLa}$ cells were observed $4 \mathrm{~h}$ postinfection, whereas $\mathrm{A} / \mathrm{E}$ lesions were rarely observed in uninfected cells $(<1 \mathrm{~A} / \mathrm{E}$ lesion per $100 \mathrm{HeLa}$ cells). When HeLa cells were infected with $\triangle$ tnaA EHEC, the number of $\mathrm{A} / \mathrm{E}$ lesions produced was about half the wild-type value $(6 \pm 2 \mathrm{~A} / \mathrm{E}$ lesions per $100 \mathrm{HeLa}$ cells). However, when $\Delta t n a \mathrm{~A}$ EHEC was cultured for $8 \mathrm{~h}$ (stationary phase) with $125 \mu \mathrm{M}$ indole before infection, the number of $\mathrm{A} / \mathrm{E}$ lesions reached about $18 \pm 3$ per $100 \mathrm{HeLa}$ cells, indicating that indole restores and promotes the induction of $\mathrm{A} / \mathrm{E}$ lesions by $\Delta$ tna $\mathrm{A}$ EHEC in HeLa cells.

\section{DISCUSSION}

Homologues of E. coli K-12 tnaA are found in many bacteria, and indole-producing bacteria are found in the human intestinal tract (DeMoss \& Moser, 1969). It was previously reported that tnaA deletion in EPEC abrogates the ability to paralyse or kill Caenorhabditis elegans (Anyanful et al., 2005). Although exogenous indole induces LEE1 expression, the reduction of virulence in the tnaA mutant was not restored by indole. On the other hand, it was recently reported that indole represses several EHEC virulence-related phenotypes (motility, biofilm formation and attachment to HeLa cells) (Bansal et al., 2007). However, the relationship between indole and type III secretion, and the formation of $\mathrm{A} / \mathrm{E}$ lesions, has not been investigated previously.

We show here that indole increases the production and secretion of EspA/B, which are T3SS-mediated translocators. This results in an increase in the formation of $\mathrm{A} / \mathrm{E}$ lesions in HeLa cells. Indole also elicits a modest induction of LEE4 transcription. We found that deletion of tnaA reduced the secretion and production of EspA/B and the formation of A/E lesions in HeLa cells (Fig. 2). All of these effects were overcome by addition of $125 \mu \mathrm{M}$ indole to O157 $\Delta$ tnaA cultures. This is a physiologically relevant indole concentration: in wild-type EHEC culture medium in the stationary phase, the indole concentration is about $465 \mu \mathrm{M}$ (Fig. 1). Therefore, the effect of tnaA deletion can be explained by the loss of indole (Fig. 2).

Experiments using a T3SS mutant, $\Delta e s c N$, showed that indole stimulates the production of EspA and EspB without secretion and the deletion of tnaA decreases cellular EspA and EspB levels (Fig. 3). However, the 
influence of indole addition and the tnaA deletion in $\Delta e s c N$ was less than that in the parent strain. We also showed that the promoter activity of espA and espB (located in the LEE4 operon) was moderately decreased by the deletion of $t n a A$, and induced by indole (Fig. 4a). The change of promoter activity was less than that of EspA and EspB protein levels. Thus, indole participates in three kinds of regulatory process of EspA/B. Among them, indole primarily controls the secretion of EspA/B. Indole also appears to control EspA and EspB translation and at the level of transcription.

We also found that deletion of $h f q$ abolishes the influence of indole on the induction of LEE4, and fully induces it even when indole is absent. These results suggest that $\mathrm{Hfq}$ tightly represses the expression of LEE4. Hfq acts as a regulator in complex with sRNAs (Gottesman, 2004), which determine the specificity of regulation. In addition, we note that the effect of $h f q$ deletion on the increase of EspA/B production is greater than that on the increase of the promoter activity (Figs $4 c, 5$ and 6). We interpret this to mean that the LEE4 operon including esp $A$ and $\operatorname{esp} B$ may be transcriptionally and post-transcriptionally regulated by Hfq-sRNA. It will be of interest to identify the hypothetical sRNA for regulation of EspA and EspB.

It was previously reported that motility-related genes and LEE operons are inversely regulated by GrlA and GrlR (Iyoda et al., 2006). Therefore, GrlA and GrlR could be considered as candidates mediating the induction of $L E E$ genes and the repression of motility by indole. However, this seems unlikely because the $L E E$ gene induction by indole is independent of Ler (Fig. 4c) whereas the regulation of LEE promoters by GrlA and GrlR depends on Ler (Iyoda et al., 2006).

In EHEC, effector proteins are delivered into host cells through EspA filaments and pores in the plasma membrane formed by EspB. Intimin, an outer-membrane protein of EHEC, then binds to Tir on the host-cell plasma membrane (Garmendia et al., 2005; Kenny et al., 1997). Although indole obviously enhances the secretion of EspA and EspB, the production of Tir and intimin (the eae gene product) is not affected (data not shown). This suggests that indole probably assists the efficient delivery of effector proteins, promoting $\mathrm{A} / \mathrm{E}$ lesion formation.

If indole is produced in stationary phase in LB medium, it is anticipated that the indole-regulated LEE4 promoter should be induced in stationary phase. The activity of this promoter in exponential phase is clearly lower than in stationary phase. However, LEE4 promoter activity of a tnaA deletion mutant in stationary phase and LEE4 in exponential phase is reasonably high (Fig. 4a, b). Therefore, we believe that indole partly contributes to the stimulation of $L E E$ gene expression in the stationary phase.

There are many recent reports that intercellular signal molecules contribute to the pathogenicity of bacteria
(Bassler, 2002). In Gram-negative bacteria, three kinds of quorum-sensing molecules (or autoinducers) (AI-1, AI-2 and AI-3) have been reported (Fuqua et al., 2001; Sperandio et al., 2001, 2003). In addition, there is evidence that indole also acts as an intercellular signal molecule during the stationary phase in E. coli and other enteric species (Kobayashi et al., 2006; Lee et al., 2007a; Wang et al., 2001). Here we show that indole enhances expression of specific virulence factors in EHEC. Previously, the relationship between intercellular signal transduction and the production of type III secretionrelated proteins was described in EHEC. A LuxR-like quorum-sensing regulator, SdiA, AI-3 and related regulatory factors (luxS, qseA and qseBC) affect the production of type III secretion-related proteins (Kanamaru et al., 2000; Sharp \& Sperandio, 2007; Sperandio et al., 1999, 2003). However, the addition of indole did not affect the transcription of the related genes (sdiA, luxS, qseA and qseBC) (data not shown) although indole activates the transcription of $s d i A$, and results in reduction of biofilm formation in E. coli $\mathrm{K}-12$ (Lee et al., 2007b). In addition, indole is produced later in the stationary phase than AI-1, AI-2 and AI-3, which are active in the early stationary phase. Therefore, the indolesensing system probably differs from these intercellular signal transduction systems.

As mentioned above, it was previously reported that indole represses several virulence-related phenotypes in EHEC (motility, biofilm formation and attachment to HeLa cells) (Bansal et al., 2007). Addition of 500-600 $\mu \mathrm{M}$ indole decreased these phenotypes whereas we showed that type III secretion-related protein production and virulence phenotypes are stimulated by indole concentrations of $125 \mu \mathrm{M}$ or more. These results may indicate that indole has dual roles in the virulence of EHEC. Judging from these observations, the virulence of EHEC seems to be tightly regulated by the concentration of indole. Indole concentration in the enteric site may change intermittently with the amount of indole-producing enteric bacteria and enteric environmental conditions. According to an in vivo study with mice, tryptophan in the food markedly affects tryptophanase, and hence presumably indole production, by enteric bacteria (Botsford \& Demoss, 1972).

To counteract bacterial infections, it is important to understand the behaviour of bacteria at their site of infection and in their habitat. Although indole is secreted by many bacteria and is abundant in the intestinal tract, its significance in bacterial physiology has remained unclear. We therefore believe that the characterization of the indole signal and the identification of an indole regulon have great interest. Moreover, interestingly, serotonin, melatonin and indole-3-acetic acid, which are typical signal molecules secreted by animal and plant cells, have an indole-like chemical structure. Therefore, it will be intriguing to investigate how these indole-like signals act on bacteria, or 
how indole affects the signal transduction in eukaryotic hosts.

\section{ACKNOWLEDGEMENTS}

We wish to thank E. Peter Greenberg for constructive comments, George M. Church for the plasmid pKO3, and Ronald W. Davis for the plasmid pNN387. We are grateful for the technical assistance in HPLC analysis provided by Nobuo Kato and Hajime Nitta of the Institute of Scientific and Industrial Research, Osaka University. H. H. was supported by a research fellowship from the Japan Society for the Promotion of Science for Young Scientists. This research was supported by a Grant-in-Aid from the Ministry of Education, Culture, Sports, Science and Technology of Japan, and CREST, Japan Science and Technology Agency, Japan.

\section{REFERENCES}

Anyanful, A., Dolan-Livengood, J. M., Lewis, T., Sheth, S., Dezalia, M. N., Sherman, M. A., Kalman, L. V., Benian, G. M. \& Kalman, D. (2005). Paralysis and killing of Caenorhabditis elegans by enteropathogenic Escherichia coli requires the bacterial tryptophanase gene. Mol Microbiol 57, 988-1007.

Bansal, T., Englert, D., Lee, J., Hegde, M., Wood, T. K. \& Jayaraman, A. (2007). Differential effects of epinephrine, norepinephrine, and indole on Escherichia coli O157:H7 chemotaxis, colonization and gene expression. Infect Immun 75, 4597-4607.

Bassler, B. L. (2002). Small talk. Cell-to-cell communication in bacteria. Cell 109, 421-424.

Blankenhorn, D., Phillips, J. \& Slonczewski, J. (1999). Acid- and base-induced proteins during aerobic and anaerobic growth of Escherichia coli revealed by two-dimensional gel electrophoresis. J Bacteriol 181, 2209-2216.

Botsford, J. L. \& Demoss, R. D. (1972). Escherichia coli tryptophanase in the enteric environment. J Bacteriol 109, 74-80.

Cantarelli, V. V., Kodama, T., Nijstad, N., Abolghait, S. K., Nada, S., Okada, M., lida, T. \& Honda, T. (2007). Tyrosine phosphorylation controls cortactin binding to two enterohaemorrhagic Escherichia coli effectors: Tir and EspFu/TccP. Cell Microbiol 9, 1782-1795.

Deeley, M. C. \& Yanofsky, C. (1982). Transcription initiation at the tryptophanase promoter of Escherichia coli K-12. J Bacteriol 151, 942 951.

DeMoss, R. D. \& Moser, K. (1969). Tryptophanase in diverse bacterial species. J Bacteriol 98, 167-171.

Di Martino, P., Fursy, R., Bret, L., Sundararaju, B. \& Phillips, R. S. (2003). Indole can act as an extracellular signal to regulate biofilm formation of Escherichia coli and other indole-producing bacteria. Can J Microbiol 49, 443-449.

Elledge, S. J. \& Davis, R. W. (1989). Position and density effects on repression by stationary and mobile DNA-binding proteins. Genes Dev 3, 185-197.

Elliott, S. J., Sperandio, V., Girón, J., Shin, S., Mellies, J., Wainwright, L., Hutcheson, S., McDaniel, T. \& Kaper, J. B. (2000). The locus of enterocyte effacement (LEE)-encoded regulator controls expression of both LEE- and non-LEE-encoded virulence factors in enteropathogenic and enterohemorrhagic Escherichia coli. Infect Immun 68, 6115-6126.

Fuqua, C., Parsek, M. R. \& Greenberg, E. P. (2001). Regulation of gene expression by cell-to-cell communication: acyl-homoserine lactone quorum sensing. Annu Rev Genet 35, 439-468.
Garmendia, J., Frankel, G. \& Crepin, V. (2005). Enteropathogenic and enterohemorrhagic Escherichia coli infections: translocation, translocation, translocation. Infect Immun 73, 2573-2585.

Gottesman, S. (2004). The small RNA regulators of Escherichia coli: roles and mechanisms. Annu Rev Microbiol 58, 303-328.

Hayashi, T., Makino, K., Ohnishi, M., Kurokawa, K., Ishii, K., Yokoyama, K., Han, C. G., Ohtsubo, E., Nakayama, K. \& other authors (2001). Complete genome sequence of enterohemorrhagic Escherichia coli $\mathrm{O} 157: \mathrm{H} 7$ and genomic comparison with a laboratory strain K-12. DNA Res 8, 11-22.

Hirakawa, H., Inazumi, Y., Masaki, T., Hirata, T. \& Yamaguchi, A. (2005). Indole induces the expression of multidrug exporter genes in Escherichia coli. Mol Microbiol 55, 1113-1126.

lyoda, S., Koizumi, N., Satou, H., Lu, Y., Saitoh, T., Ohnishi, M. \& Watanabe, H. (2006). The GrlR-GrlA regulatory system coordinately controls the expression of flagellar and LEE-encoded type III protein secretion systems in enterohemorrhagic Escherichia coli. J Bacteriol 188, 5682-5692.

Kanamaru, K., Kanamaru, K., Tatsuno, I., Tobe, T. \& Sasakawa, C. (2000). SdiA, an Escherichia coli homologue of quorum-sensing regulators, controls the expression of virulence factors in enterohaemorrhagic Escherichia coli O157:H7. Mol Microbiol 38, 805-816.

Karmali, M. A. (1989). Infection by verocytotoxin-producing Escherichia coli. Clin Microbiol Rev 2, 15-38.

Kawamura-Sato, K., Shibayama, K., Horii, T., limuma, Y., Arakawa, Y. \& Ohta, M. (1999). Role of multiple efflux pumps in Escherichia coli in indole expulsion. FEMS Microbiol Lett 179, 345-352.

Kenny, B., DeVinney, R., Stein, M., Reinscheid, D. J., Frey, E. A. \& Finlay, B. B. (1997). Enteropathogenic E. coli (EPEC) transfers its receptor for intimate adherence into mammalian cells. Cell 91, 511520.

Kobayashi, A., Hirakawa, H., Hirata, T., Nishino, K. \& Yamaguchi, A. (2006). The growth phase-dependent expression of drug exporters in Escherichia coli and its contribution to the drug tolerance. J Bacteriol 188, 5693-5703.

Kodama, T., Akeda, Y., Kono, G., Takahashi, A., Imura, K., lida, T. \& Honda, T. (2002). The EspB protein of enterohaemorrhagic Escherichia coli interacts directly with alpha-catenin. Cell Microbiol 4, 213-222.

Lee, J., Bansal, T., Jayaraman, A., Bentley, W. \& Wood, T. K. (2007a). Enterohemorrhagic Escherichia coli biofilms are inhibited by 7hydroxyindole and stimulated by isatin. Appl Environ Microbiol 73, 4100-4109.

Lee, J., Jayaraman, A. \& Wood, T. K. (2007b). Indole is an interspecies biofilm signal mediated by SdiA. BMC Microbiol 7, 42.

Link, A. J., Phillips, D. \& Church, G. M. (1997). Methods for generating precise deletions and insertions in the genome of wild-type Escherichia coli: application to open reading frame characterization. J Bacteriol 179, 6228-6237.

McDaniel, T. K. \& Kaper, J. B. (1997). A cloned pathogenicity island from enteropathogenic Escherichia coli confers the attaching and effacing phenotype on E. coli K-12. Mol Microbiol 23, 399-407.

Miller, J. H. (1992). A Short Course in Molecular Genetics. Cold Spring Harbor, NY: Cold Spring Harbor Laboratory.

Nataro, J. P. \& Kaper, J. B. (1998). Diarrheagenic Escherichia coli. Clin Microbiol Rev 11, 142-201.

Sharp, F. C. \& Sperandio, V. (2007). QseA directly activates transcription of LEE1 in enterohemorrhagic Escherichia coli. Infect Immun 75, 2432-2440.

Sperandio, V., Mellies, J. L., Nguyen, W., Shin, S. \& Kaper, J. B. (1999). Quorum sensing controls expression of the type III secretion 
gene transcription and protein secretion in enterohemorrhagic and enteropathogenic Escherichia coli. Proc Natl Acad Sci U S A 96, 1519615201.

Sperandio, V., Torres, A. G., Giron, J. A. \& Kaper, J. B. (2001). Quorum sensing is a global regulatory mechanism in enterohemorrhagic Escherichia coli O157: H7. J Bacteriol 183, 5187-5197.
Sperandio, V., Torres, A. G., Jarvis, B., Nataro, J. P. \& Kaper, J. B. (2003). Bacteria-host communication: the language of hormones. Proc Natl Acad Sci U S A 100, 8951-8956.

Wang, D., Ding, X. \& Rather, P. N. (2001). Indole can act as an extracellular signal in Escherichia coli. J Bacteriol 183, 4210-4216.

Edited by: B. Kenny 